\title{
FIAT MONEY AND THE VALUE OF BINDING PORTFOLIO CONSTRAINTS
}

Autor: Mário Páscoa, Myrian Petrassi, Juan Pablo Torres-Martínez

Santiago, Agosto 2008

La serie de Documentos de Trabajo (SDT) del Departamento de Economía de la Universidad de Chile en versión PDF puede descargarse en la dirección electrónica www.econ.uchile.cl/SDT . Para contactar al editor ejecutivo de SDT remitirse a sdt@econ.uchile.cl 


\title{
Serie Documentos de Trabajo \\ N 283
}

\section{Fiat money and the value of binding portfolio constraints}

\author{
Mário Páscoa \\ Myrian Petrassi \\ Juan Pablo Torres-Martínez
}

\begin{abstract}
It is well known that, under uniform impatience, positive net supply assets are free of bubbles for non-arbitrage kernel deflators that yield finite present values of wealth. However, this does not mean that prices cannot be above the series of deflated dividends for the deflators given by the agents' marginal rates of substitution, which also yield finite present values of wealth. In particular, binding no-short-sales constraints lead to positive prices of fiat money. Also, monetary equilibria are Pareto improvements but they are still inefficient.
\end{abstract}

\section{Keywords:}

Binding debt constraints, Fundamental value of money, Asset pricing bubbles. 


\title{
FIAT MONEY AND THE VALUE OF BINDING PORTFOLIO CONSTRAINTS
}

\author{
MÁRIO R. PÁSCOA, MYRIAN PETRASSI AND JUAN PABLO TORRES-MARTÍNEZ
}

\begin{abstract}
It is well known that, under uniform impatience, positive net supply assets are free of bubbles for non-arbitrage kernel deflators that yield finite present values of wealth. However, this does not mean that prices cannot be above the series of deflated dividends for the deflators given by the agents' marginal rates of substitution, which also yield finite present values of wealth. In particular, binding no-short-sales constraints lead to positive prices of fiat money. Also, monetary equilibria are Pareto improvements but they are still inefficient.
\end{abstract}

KEYwORDs: Binding debt constraints, Fundamental value of money, Asset pricing bubbles.

JEL classification: D50, D52.

\section{INTRODUCTION}

The uniform impatience assumption (see Hernandez and Santos (1996, Assumption C.3) or Magill and Quinzii (1996, Assumptions B2, B4)), together with borrowing constraints, is a usual requirement for existence of equilibrium in economies with infinite lived agents. This condition is satisfied whenever preferences are separable over time and across states so long as (i) the inter-temporal discounted factor is constant, (ii) individual endowments are uniformly bounded away from zero, and (iii) aggregate endowments are uniformly bounded from above.

The assumption of uniform impatience has important implications for asset pricing as it rules out speculation in assets in positive net supply for deflator processes in the non-arbitrage pricing kernel, yielding finite present values of aggregate wealth, as Santos and Woodford (1997) showed. The well-known example of a positive price of fiat money by Bewley (1980) highlighted the importance of the finiteness of the present value of aggregate wealth.

What happens if we use as deflators the agents' inter-nodes marginal rates of substitution? These deflators may fail to be in the asset pricing kernel when some portfolio constraints are binding. ${ }^{1}$ For these Kuhn-Tucker deflators, assets in positive net supply may be priced above the series of deflated dividends and the difference may be due to the presence of shadow prices rather than due

Revised version: July, 2008.

Previous versions appeared as PUC-Rio's discussion papers under the titles: "On the role of debt constraints in monetary equilibrium" and "Welfare improving debt constraints". J.P.Torres-Martínez acknowledges support from CNPq-Brazil through project 307554/2004-0. M. Petrassi wants to disclaim that the views expressed herein are not necessarily those of the Central Bank of Brazil. As visões expressas no trabalho não são necessariamente as do Banco Central do Brasil.

${ }^{1}$ Notice that under inequality constraints on portfolios, non-arbitrage (from one node to its immediate successors) is equivalent to the existence of a positive vector of state prices solving a linear system of inequalities relating asset prices and returns (see Jouini and Kallal (1995) or Araujo, Fajardo and Páscoa (2005)). The state prices that make all inequalities hold as equalities constitute the kernel of the linear operator that defines the system, but there may be other solutions (for example, those given by the Kuhn-Tucker multiplers). 
to a bubble. Gimenez (2005) already made this comment and Araujo, Pascoa and Torres-Martinez (2007) worked along these lines when addressing the pricing of long-lived collateralized assets.

To consider a simple and provocative case, we look, as in Bewley (1980), at economies with a single asset, paying no dividends and in positive net supply. As usual, we call this asset fiat money (or simply money), although we are quite aware that we are just looking at its role as a store of value, i.e. as an instrument to transfer wealth across time and states of nature. In this context and under uniform impatience, we show that money can and will only be positively valued as a result of agents' desire to take short positions that they can not. That is, under uniform impatience, the positive price of money must be due to the presence of shadow prices of binding constraints.

This result does not collide with the example by Bewley (1980) or the results by Santos and Woodford (1997). It complements these results. Under uniform impatience, a positive price of money implies that the present value of aggregate wealth must be infinite for any deflator in the asset pricing kernel. Without uniform impatience, it would only imply an infinite supremum for the present value of aggregate wealth, when all deflators in that kernel are considered. However, for any Kuhn-Tucker deflator process of a certain agent, the present value of the endowments of this agent is finite, but this deflator may fail to be in the asset pricing kernel (when this agent has binding portfolio constraints) or the present value of aggregate wealth may fail to be finite (when the deflator is not the same for all agents and uniform impatience does not hold).

In Bewley's (1980) example, the two uniformly impatient agents were not allowed to take short positions and the economy had no uncertainty. The positive price of money was a bubble for the unique deflator process in the asset pricing kernel and for this deflator the present value of aggregate wealth was infinite. However, the no-short-sales constraint was binding infinitely often. Thus, for the Kuhn-Tucker deflator process of each agent, the fundamental value of money was positive, consisting of the shadow prices of debt constraints.

Hence, we obtain a result that may seem surprising: credit frictions create room for welfare improvements through transfers of wealth that become possible when money has a positive price. However, monetary equilibria are always Pareto inefficient. Otherwise, by definition, agents' rates of inter-temporal substitution would coincide. However, as money is in positive net supply, at least one agent must go long, having a zero shadow price. Thus, the shadow prices of all agents should be zero and, therefore, the price of money could not be positive.

To clarify our results, we prove that when money has a positive value, there exists a deflator, but not one of the Kuhn-Tucker deflators, under which the discounted value of aggregated wealth is infinite and a pure bubble appears. That is, in our framework (that includes Bewley (1980) model) it is always possible to interpret monetary equilibrium as a bubble. However, when we focus on Kuhn-Tucker multipliers - deflators that make financial Euler conditions compatible with physical Euler conditions - the positive price of money is always a consequence of a positive fundamental value.

We close the paper with an example of a stochastic economy that does not satisfy the uniform impatience assumption. Money is positive valued in equilibrium, although shadow prices of debt 
constraints are zero. For the Kuhn-Tucker deflator processes of both agents, aggregate wealth has a finite present value.

Our main mathematical tool is a duality approach to dynamic programming problems that was already used in the context of long-lived collateralized assets by Araujo, Páscoa and Torres-Martinez (2007). This approach allows us to characterize non-interior solutions and the respective KuhnTucker multiplier processes. A recent related paper by Rincón-Zapatero and Santos (2007) addresses the uniqueness of this multiplier process and the differentiability of the value function, without imposing the usual interiority assumptions.

The paper is organized as follows. Section 2 characterizes uniform impatience. Section 3 presents the basic model. In Section 4, we develop the necessary mathematical tools: a duality theory of individual optimization. In Section 5 we define the concepts of fundamental value of money and asset pricing bubbles. Finally, Section 6 presents the results on monetary equilibria and Section 7 an example of monetary equilibrium in an economy without uniform impatience. Some proofs are left to the Appendix.

\section{Characterizing Uniform impatience When Utilities ARE SEPARABle}

In this section, we recall the assumption of uniform impatience and characterize it for separable utilities in terms of inter-temporal discount factors. As a consequence, we show that the uniform impatience assumption does not hold for agents with hyperbolic inter-temporal discounting (see Laibson (1998)).

Consider an infinite horizon discrete time economy where the set of dates is $\{0,1, \ldots\}$ and there is no uncertainty at $t=0$. Given a history of realizations of the states of nature for the first $t-1$ dates, with $t \geq 1, \bar{s}_{t}=\left(s_{0}, \ldots, s_{t-1}\right)$, there is a finite set $S\left(\bar{s}_{t}\right)$ of states that may occur at date $t$. A vector $\xi=\left(t, \bar{s}_{t}, s\right)$, where $t \geq 1$ and $s \in S\left(\bar{s}_{t}\right)$, is called a node. The only node at $t=0$ is denoted by $\xi_{0}$. Let $D$ be the event-tree, i.e., the set of all nodes.

Given $\xi=\left(t, \bar{s}_{t}, s\right)$ and $\mu=\left(t^{\prime}, \bar{s}_{t^{\prime}}, s^{\prime}\right)$, we say that $\mu$ is a successor of $\xi$, and we write $\mu \geq \xi$, if $t^{\prime} \geq t$ and $\bar{s}_{t^{\prime}}=\left(\bar{s}_{t}, s, \ldots\right)$. We write $\mu>\xi$ to say that $\mu \geq \xi$ but $\mu \neq \xi$ and we denote by $t(\xi)$ the date associated with a node $\xi$. Let $\xi^{+}=\{\mu \in D:(\mu \geq \xi) \wedge(t(\mu)=t(\xi)+1)\}$ be the set of immediate successors of $\xi$. The (unique) predecessor of $\xi>\xi_{0}$ is denoted by $\xi^{-}$and $D(\xi):=\{\mu \in D: \mu \geq \xi\}$ is the sub-tree with root $\xi$.

At any node $\xi \in D$, a finite set of perishable commodities is available for trade, $L$. There is a finite set of infinite-lived agents, $H$. Each agent $h \in H$ has at any $\xi \in D$ a physical endowment $w^{h}(\xi) \in \mathbb{R}_{+}^{L}$ and has preferences over consumption plans which are represented by a function $U^{h}: \mathbb{R}_{+}^{L \times D} \rightarrow \mathbb{R}_{+} \cup\{+\infty\}$. Aggregated physical endowments at a node $\xi$ are given by $W_{\xi} \in \mathbb{R}_{++}^{L}$.

Assumption 1 (Separability of Preferences). Let $U^{h}(x):=\sum_{\xi \in D} u^{h}(\xi, x(\xi))$, where for any $\xi \in D, u^{h}(\xi, \cdot): \mathbb{R}_{+}^{L} \rightarrow \mathbb{R}_{+}$is a continuous, concave and strictly increasing function. Also, $\sum_{\xi \in D} u^{h}\left(\xi, W_{\xi}\right)$ is finite. 
Assumption 2 (Uniform impatience). There are $\pi \in[0,1)$ and $(v(\mu) ; \mu \in D) \in \mathbb{R}_{+}^{D \times L}$ such that, given a consumption plan $(x(\mu) ; \mu \in D)$, with $0 \leq x(\mu) \leq W_{\mu}$, for any $h \in H$, we have

$$
u^{h}(\xi, x(\xi)+v(\xi))+\sum_{\mu>\xi} u^{h}\left(\mu, \pi^{\prime} x(\mu)\right)>\sum_{\mu \geq \xi} u^{h}(\mu, x(\mu)), \quad \forall \xi \in D, \quad \forall \pi^{\prime} \geq \pi .
$$

Moreover, there is $\delta^{h}>0$ such that, $w^{h}(\xi) \geq \delta^{h} v(\xi), \forall \xi \in D$.

The requirements of impatience above depend on both preferences and physical endowments. As particular cases we obtain the assumptions imposed by Hernandez and Santos (1996) and Magill and Quinzii (1994). Indeed, in Hernandez and Santos (1996), for any $\mu \in D, v(\mu)=W_{\mu}$. Also, since in Magill and Quinzii $(1994,1996)$ initial endowments are uniformly bounded away from zero by an interior bundle $\underline{w}^{h} \in \mathbb{R}_{+}^{L}$, they suppose that $v(\mu)=(1,0, \ldots, 0), \forall \mu \in D$.

Our characterization of uniform impatience is,

Proposition 1. Suppose that Assumption 1 holds, that $\left(W_{\xi} ; \xi \in D\right)$ is a bounded plan and that there is $\underline{w}^{h} \in \mathbb{R}_{+}^{L} \backslash\{0\}$ such that, $w^{h}(\xi) \geq \underline{w}^{h}, \forall \xi \in D$. Moreover, there exists a function $u^{h}: \mathbb{R}_{+}^{L} \rightarrow \mathbb{R}_{+}$ such that, for any $\xi \in D, u^{h}(\xi, \cdot) \equiv \beta_{t(\xi)}^{h} \rho^{h}(\xi) u^{h}(\cdot)$, where $\beta_{t(\xi)}^{h}>0, \rho^{h}(\xi)=\sum_{\mu \in \xi^{+}} \rho^{h}(\mu)$ and $\rho^{h}\left(\xi_{0}\right)=1$. For each $t \geq 0$, let $s_{t}^{h}=\frac{1}{\beta_{t}^{h}} \sum_{r=t+1}^{+\infty} \beta_{r}^{h}$. Then, the function $U^{h}$ satisfies uniform impatience (Assumption 2) if and only if $\left(s_{t}^{h}\right)_{t \geq 0}$ is bounded.

Proof. Assume that $\left(W_{\xi} ; \xi \in D\right)$ is a bounded plan. That is, there is $\bar{W} \in \mathbb{R}_{+}^{L}$ such that, $W_{\xi} \leq \bar{W}, \forall \xi \in D$. If $\left(s_{t}^{h}\right)_{t \geq 0}$ is bounded, then there exists $\bar{s}^{h}>0$ such that, $s_{t}^{h} \leq \bar{s}^{h}$, for each $t \geq 0$. Also, since $\mathbb{F}:=\left\{x \in \mathbb{R}_{+}^{L}: x \leq \bar{W}\right\}$ is compact, the continuity of $u^{h}$ assures that there is $\pi \in(0,1)$ such that $u^{h}(x)-u^{h}\left(\pi^{\prime} x\right) \leq \frac{u^{h}\left(\bar{W}+\underline{w}^{h}\right)-u^{h}(\bar{W})}{2 \bar{s}^{h}}, \forall x \in \mathbb{F}, \forall \pi^{\prime} \geq \pi$. Thus, uniform impatience follows by choosing $\delta=1$ and $v(\xi)=\underline{w}^{h}, \forall \xi \in D$. Indeed, given a plan $(x(\mu) ; \mu \in D) \in \mathbb{R}_{+}^{L \times D}$ such that, $x(\mu) \leq W_{\mu} \forall \mu \in D$, the concavity of $u^{h}$ assures that, for any $\xi \in D$ and $\pi^{\prime} \geq \pi$,

$$
\begin{aligned}
\sum_{\mu>\xi} \beta_{t(\mu)}^{h} \rho^{h}(\mu) u^{h}(x(\mu))-\sum_{\mu>\xi} \beta_{t(\mu)}^{h} \rho^{h}(\mu) u^{h}\left(\pi^{\prime} x(\mu)\right) & \leq \frac{\beta_{t(\xi)}^{h} s_{t}}{2 \bar{s}^{h}} \rho^{h}(\xi)\left(u^{h}\left(\bar{W}+\underline{w}^{h}\right)-u^{h}(\bar{W})\right) \\
& <\beta_{t(\xi)}^{h} \rho^{h}(\xi) u^{h}(x(\xi)+v(\xi))-\beta_{t(\xi)}^{h} \rho^{h}(\xi) u^{h}(x(\xi)) .
\end{aligned}
$$

Reciprocally, suppose that uniform impatience property holds. Then, given $(x(\mu) ; \mu \in D) \in$ $\mathbb{R}_{+}^{L \times D}$ such that, $x(\mu) \leq W_{\mu}$, for all $\mu \in D$, there are $\left(\pi, \delta^{h}\right) \in[0,1) \times \mathbb{R}_{++}$and $(v(\mu) ; \mu \in D) \mathbb{R}_{+}^{D \times L}$ satisfying, for any $\xi \in D, w^{h}(\xi) \geq \delta^{h} v(\xi)$, such that, for any $\xi \in D$,

$$
\frac{1}{\beta_{t(\xi)}^{h} \rho^{h}(\xi)}\left[\sum_{\mu>\xi} \beta_{t(\mu)}^{h} \rho^{h}(\mu) u^{h}(x(\mu))-\sum_{\mu>\xi} \beta_{t(\mu)}^{h} \rho^{h}(\mu) u^{h}(\pi x(\mu))\right]<u^{h}(x(\xi)+v(\xi))-u^{h}(x(\xi)) .
$$

It follows that, for any node $\xi$,

$$
\frac{1}{\beta_{t(\xi)}^{h} \rho^{h}(\xi)}\left[\sum_{\mu>\xi} \beta_{t(\mu)}^{h} \rho^{h}(\mu) u^{h}(\underline{w})-\sum_{\mu>\xi} \beta_{t(\mu)}^{h} \rho^{h}(\mu) u^{h}(\pi \underline{w})\right]<u^{h}\left(\left(1+\frac{1}{\delta^{h}}\right) \bar{W}\right) .
$$


Therefore, we conclude that, for any $\xi \in D$,

$$
\frac{1}{\beta_{t(\xi)}^{h}}\left(u^{h}\left(\underline{w}^{h}\right)-u^{h}\left(\pi \underline{w}^{h}\right)\right) \sum_{t=t(\xi)+1}^{+\infty} \beta_{t}^{h}<u^{h}\left(\left(1+\frac{1}{\delta^{h}}\right) \bar{W}\right),
$$

which implies that the sequence $\left(s_{t}^{h}\right)_{t \geq 0}$ is bounded.

Under the conditions of Proposition 1, if inter-temporal discount factors are constant, i.e. $\exists c^{h} \in$ $\mathbb{R}_{++}: \frac{\beta_{t(\xi)+1}^{h}}{\beta_{t(\xi)}^{h}}=c^{h}, \forall \xi \in D$, then $c^{h}<1$ and $s_{t}^{h}=\frac{c^{h}}{1-c^{h}}$, for each $t \geq 0$. In this case, the utility function $U^{h}$ satisfies the uniform impatience condition.

However, even with bounded plans of endowments, uniform impatience is a restrictive condition when inter-temporal discount factors are time varying. For instance, if we consider hyperbolic intertemporal discount factors, that is, $\beta_{t}^{h}=(1+a t)^{-\frac{b}{a}}$, where $b>a>0$, then the function $U^{h}$, as defined in the statement of Proposition 1, satisfies Assumption 1 and the sequence $s_{t}^{h}$ goes to infinity as $t$ increases. Therefore, in this case, uniform impatience does not hold.

\section{A MONETARY MODEL WITH UNIFORM IMPATIENCE AGENTS}

We assume that there is only one asset, money, that can be traded along the event-tree. Although this security does not deliver any payment, it can be used to make inter-temporal transfers. Let $q=(q(\xi) ; \xi \in D)$ be the plan of monetary prices. We assume that money is in positive net supply that does not disappear from the economy neither depreciates. Denote money endowments by $e^{h}(\xi) \in \mathbb{R}_{+}$, at each $\xi \in D$ (we often suppose null endowments except at the initial node).

Let $z^{h}(\xi)$ denote the quantity of money that $h$ negotiates at $\xi$. Thus, if $z^{h}(\xi)>0$, she buys the asset, otherwise, she short sales money making future promises (although we will constrain these short-sales or even rule them out).

Let $p(\xi):=(p(\xi, l) ; l \in L)$ be the commodity price at $\xi \in D$ and $p=(p(\xi) ; \xi \in D)$.

Given prices $(p, q)$, let $B^{h}(p, q)$ be the choice set of agent $h \in H$, that is, the set of plans $(x, z):=((x(\xi), z(\xi)) ; \xi \in D) \in \mathbb{R}_{+}^{D \times L} \times \mathbb{R}^{D}$, such that, at any $\xi \in D$, the following budget and debt constraints hold,

$$
\begin{aligned}
g_{\xi}^{h}\left(y^{h}(\xi), y^{h}\left(\xi^{-}\right) ; p, q\right):=p(\xi)\left(x^{h}(\xi)-w^{h}(\xi)\right)+q(\xi)\left(z^{h}(\xi)-e^{h}(\xi)-z^{h}\left(\xi^{-}\right)\right) & \leq 0 \\
q(\xi) z^{h}(\xi)+p(\xi) M & \geq 0
\end{aligned}
$$

where $y^{h}(\xi)=\left(x^{h}(\xi), z^{h}(\xi)\right), y^{h}\left(\xi_{0}^{-}\right):=\left(x^{h}\left(\xi_{0}^{-}\right), z^{h}\left(\xi_{0}^{-}\right)=0\right.$ and $M \in \mathbb{R}_{+}^{L}$. Note that short sales of money are bounded by an exogenous debt constraints.

Agent's $h$ individual problem is to choose a plan $y^{h}=\left(x^{h}, z^{h}\right)$ in $B^{h}(p, q)$ in order to maximize her utility function $U^{h}: \mathbb{R}_{+}^{L \times D} \rightarrow \mathbb{R}_{+} \cup\{+\infty\}$.

\section{DEFINITION 1 .}

An equilibrium for our economy is given by a vector of prices $(p, q)$ jointly with individual plans $\left(\left(x^{h}, z^{h}\right) ; h \in H\right)$, such that,

(a) For each $h \in H$, the plan $\left(x^{h}, z^{h}\right) \in B^{h}(p, q)$ is optimal at prices $(p, q)$. 
(b) Physical and asset markets clear,

$$
\sum_{h \in H}\left(x^{h}(\xi) ; z^{h}(\xi)\right)=\left(W_{\xi}, \sum_{h \in H}\left(e^{h}(\xi)+z^{h}\left(\xi^{-}\right)\right)\right) .
$$

Note that, a pure spot market equilibrium, i.e. an equilibrium with zero monetary price, always exists provided that preferences satisfy Assumption 1 above.

\section{Duality theory For INDividual optimization}

In this section, we determine necessary and sufficient conditions for individual optimality.

Some previous definitions and notations are necessary. By normalization, we assume that prices $(p, q)$ belong to $\mathbb{P}:=\left\{(p, q) \in \mathbb{R}_{+}^{L \times D} \times \mathbb{R}_{+}^{D}:(p(\xi), q(\xi)) \in \Delta^{\# L+1}, \forall \xi \in D\right\}$, where, for each $m>0$, the simplex $\Delta^{m}:=\left\{z=\left(z_{1}, \ldots, z_{m}\right) \in \mathbb{R}_{+}^{m}: \sum_{k=1}^{m} z_{k}=1\right\}$. Given a concave function $f: X \subset \mathbb{R}^{L} \rightarrow \mathbb{R} \cup\{-\infty\}$ the super-differential at $x \in X$ is defined as the set of vectors $p \in \mathbb{R}^{L}$ such that, for all $x^{\prime} \in X, f\left(\xi, x^{\prime}\right)-f(\xi, x) \leq p\left(x^{\prime}-x\right)$. The set of nodes with date $T$ in $D(\xi)$ is denoted by $D_{T}(\xi)$, and $D^{T}(\xi)=\bigcup_{k=t(\xi)}^{T} D_{k}(\xi)$ denotes the set of successors of $\xi$ with date less than or equal to $T$. When $\xi=\xi_{0}$ notations above will be shorten to $D_{T}$ and $D^{T}$.

\section{DEFINITION 2.}

Given $(p, q) \in \mathbb{P}$ and $y^{h}=\left(x^{h}, z^{h}\right) \in B^{h}(p, q)$, we say that $\left(\gamma^{h}(\xi) ; \xi \in D\right) \in \mathbb{R}_{+}^{D}$ constitutes a family of Kuhn-Tucker multipliers (associated to $y^{h}$ ) if there exist, for each $\xi \in D$, super-gradients $u^{\prime}(\xi) \in \partial u^{h}\left(\xi, x^{h}(\xi)\right)$ such that,

(a) For every $\xi \in D, \gamma^{h}(\xi) g_{\xi}^{h}\left(y^{h}(\xi), y^{h}\left(\xi^{-}\right) ; p, q\right)=0$.

(b) The following Euler conditions hold,

$$
\begin{aligned}
\gamma^{h}(\xi) p(\xi) & \geq u^{\prime}(\xi), \\
\gamma^{h}(\xi) p(\xi) x^{h}(\xi) & =u^{\prime}(\xi) x^{h}(\xi), \\
\gamma^{h}(\xi) q(\xi) & \geq \sum_{\mu \in \xi^{+}} \gamma^{h}(\mu) q(\mu),
\end{aligned}
$$

where the last inequality is strict only if the associated debt constraint is binding at $\xi$.

(c) The following transversality condition holds: $\lim \sup _{T \rightarrow+\infty} \sum_{\xi \in D_{T}} \gamma^{h}(\xi) q(\xi) z^{h}(\xi) \leq 0$.

Since we only know that, for any plan $(p, q) \in \mathbb{P}$, the choice set $B^{h}(p, q)$ belongs to $\mathbb{R}_{+}^{D \times L} \times \mathbb{R}^{D}$, it is not obvious that a plan of Kuhn-Tucker multipliers will exist. Thus, we need to develop a duality theory. As individual admissible plans are determined by countably many inequalities, we will construct Kuhn-Tucker multipliers using the Kuhn-Tucker Theorem for Euclidean spaces.

First of all, we want to note that, when Kuhn-Tucker multipliers exist and are used as intertemporal deflators, the discounted value of individual endowments is finite. 


\section{Proposition 2. (Finite discounted VAlue of individual ENDOWMENTS)}

Fix a plan $(p, q) \in \mathbb{P}$ and $y^{h}=\left(x^{h}, z^{h}\right) \in B^{h}(p, q)$ such that $U^{h}\left(x^{h}\right)<+\infty$. If Assumption 1 holds then for any family of Kuhn-Tucker multipliers associated to $y^{h},\left(\gamma^{h}(\xi) ; \xi \in D\right)$, we have $\sum_{\xi \in D} \gamma^{h}(\xi)\left(p(\xi) w^{h}(\xi)+q(\xi) e^{h}(\xi)\right)<+\infty$.

\section{Proposition 3. (NECESSARY CONDITIONS FOR INDIVIDUAL OPTIMALITY)}

Fix a plan $(p, q) \in \mathbb{P}$ and $y^{h}=\left(x^{h}, z^{h}\right) \in B^{h}(p, q)$ such that $U^{h}\left(x^{h}\right)<+\infty$. If Assumption 1 holds and $y^{h}$ is an optimal allocation for agent $h \in H$ at prices $(p, q)$, then there exists a family of Kuhn-Tucker multipliers associated to $y^{h}$.

As the next corollary shows, our transversality condition (see Definition 2) is equivalent to the requirement imposed by Magill and Quinzii (1996), provided that either short sales were avoided or individual endowments were uniformly bounded away from zero.

\section{Corollary.}

Fix $(p, q) \in \mathbb{P}$. Under Assumption 1, given $h \in H$ suppose that either $M=0$ or there exists $\underline{w} \in \mathbb{R}_{++}^{L}$ such that, at any $\xi \in D, w^{h}(\xi) \geq \underline{w}$. If $y^{h}$ is an optimal allocation for agent $h$ at prices $(p, q)$, then for any plan of Kuhn-Tucker multipliers associated to $y^{h},\left(\gamma^{h}(\xi)\right)_{\xi \in D}$, we have,

$$
\lim _{T \rightarrow+\infty} \sum_{\xi \in D_{T}} \gamma^{h}(\xi) q(\xi) z^{h}(\xi)=0
$$

ProOF. Let $\left(\gamma^{h}(\xi)\right)_{\xi \in D}$ be a plan of Kuhn-Tucker multipliers associated to $y^{h}$. We know that the transversality condition of Definition 2 holds. On the other hand, it follows directly from the debt constraint that,

$$
\sum_{\xi \in D_{T}} \gamma^{h}(\xi) q(\xi) z^{h}(\xi) \geq-\sum_{\xi \in D_{T}} \gamma^{h}(\xi) p(\xi) M \geq-\left(\max _{l \in L} M_{l}\right) \sum_{\xi \in D_{T}} \gamma^{h}(\xi)\|p(\xi)\|_{\Sigma} .
$$

Therefore, when $M=0$ we obtain the result. Alternatively, assume that for any $\xi \in D, w^{h}(\xi) \geq \underline{w}$. Since by Proposition 2, the $\operatorname{sum} \sum_{\xi \in D} \gamma^{h}(\xi) p(\xi) w^{h}(\xi)$ is well defined and finite, we have that $\sum_{\xi \in D} \gamma^{h}(\xi)\|p(\xi)\|_{\Sigma}<+\infty$. Thus, $\liminf \operatorname{in}_{T \rightarrow+\infty} \sum_{\xi \in D_{T}} \gamma^{h}(\xi) q(\xi) z^{h}(\xi) \geq 0$ which implies, using the transversality condition of Definition 2, that $\lim _{T \rightarrow+\infty} \sum_{\xi \in D_{T}} \gamma^{h}(\xi) q(\xi) z^{h}(\xi)=0$.

We end this section with a result that determines sufficient requirements to assure that a plan of consumption and portfolio allocations is individually optimal. Note that the result below will assure that, when either short-sales are avoided - the Bewley(1980) model—, or individual endowments are uniformly bounded away from zero, a budget feasible plan is individually optimal if and only if there exists a family of Kuhn-Tucker multipliers associated with it. 


\section{Proposition 4. (SUFFICIENT CONDITIONS FOR INDIVIDUAL OPTIMALITy)}

Fix a plan $(p, q) \in \mathbb{P}$. Under Assumption 1, suppose that given $y^{h}=\left(x^{h}, z^{h}\right) \in B^{h}(p, q)$ there exists a family of Kuhn-Tucker multipliers $\left(\gamma^{h}(\xi) ; \xi \in D\right)$ associated to $y^{h}$. If

$$
\lim _{T \rightarrow+\infty} \sum_{\xi \in D_{T}} \gamma^{h}(\xi) p(\xi) M=0
$$

then $y^{h}$ is an optimal allocation for agent $h$ at prices $(p, q)$.

\section{Frictions induCED By DEBT CONSTRAints, FUndAMENTAL VALUES AND BUbBles}

In a frictionless world, that is, where debt constraints are non saturated, there are two (equivalent) definitions of the fundamental value of an asset. The fundamental value is either (1) equal to the discounted value of future deliveries that an agent will receive for one unit of the asset that she buys and keeps forever; or (2) equal to the discounted value of rental services, which coincides with the value of deliveries, given the absence of any friction associated to debt constraint.

These concepts do not coincide when frictions are allowed. Thus, we adopt the second definition, that internalizes the role that money has: it allows for inter-temporal transfers, although its deliveries are zero.

\section{Proposition 5. (Non-existence of negative Bubbles)}

Under Assumption 1, given an equilibrium $\left[(p, q) ;\left(\left(x^{h}, z^{h}\right) ; h \in H\right)\right]$, at each node $\xi \in D, q(\xi) \geq$ $F\left(\xi, q, \gamma^{h}\right)$, where $\gamma^{h}:=\left(\gamma^{h}(\xi) ; \xi \in D\right)$ denotes the agent's $h$ plan of Kuhn-Tucker multipliers and

$$
F\left(\xi, q, \gamma^{h}\right):=\frac{1}{\gamma^{h}(\xi)} \sum_{\mu \in D(\xi)}\left(\gamma^{h}(\mu) q(\mu)-\sum_{\nu \in \mu^{+}} \gamma^{h}(\nu) q(\nu)\right),
$$

is the fundamental value of money at $\xi \in D$.

Note that the rental services that one unit of money gives at $\mu \in D$ are equal to $q(\mu)-$ $\sum_{\nu \in \mu^{+}} \frac{\gamma^{h}(\nu)}{\gamma^{h}(\mu)} q(\mu)$. Thus, the fundamental value of money at a node $\xi$, as was defined above, coincides with the discounted value of (unitary) future rental services.

On the other hand, under Assumption 1, it follows from Propositions 3 and 5 that, given an equilibrium $\left[(p, q) ;\left(\left(x^{h}, z^{h}\right) ; h \in H\right)\right]$, there are, for each agent $h \in H$, Kuhn-Tucker multipliers $\left(\gamma^{h}(\xi) ; \xi \in D\right)$, such that,

$$
q(\xi)=F\left(\xi, q, \gamma^{h}\right)+\lim _{T \rightarrow+\infty} \sum_{\{\mu \geq \xi: t(\mu)=T\}} \frac{\gamma^{h}(\mu)}{\gamma^{h}(\xi)} q(\mu),
$$

where the second term in the right hand side is called the bubble component of $q(\xi)$. When $q(\xi)>$ $F\left(\xi, q, \gamma^{h}\right)$ we say that fiat money has a bubble at $\xi$ under $\gamma^{h}$.

Finally, we say that debt constraints induce frictions over agent $h$ in $\tilde{D} \subset D$ if the plan of shadow prices $\left(\eta^{h}(\mu) ; \mu \in \tilde{D}\right)$ that is defined implicitly, at each $\mu \in \tilde{D}$, by the conditions:

$$
\begin{aligned}
0 & =\eta^{h}(\mu)\left(q(\mu) z^{h}(\mu)+p(\mu) M\right), \\
\gamma^{h}(\mu) q(\mu) & =\sum_{\nu \in \mu^{+}} \gamma^{h}(\nu) q(\nu)+\eta^{h}(\mu) q(\mu),
\end{aligned}
$$


is different from zero.

\section{Characterizing monetary Equilibria}

Let us see under what conditions can we have equilibria with positive price of money, also called monetary equilibria. We assume that agents are uniformly impatient, that is, Assumption 2 holds.

\section{Theorem.}

Under Assumptions 1 and 2, for any equilibrium $\left[(p, q) ;\left(\left(x^{h}, z^{h}\right) ; h \in H\right)\right]$ we have that,

(1) If $q(\xi)>0$ then debt constraints induce frictions over each agent in $D(\xi)$.

(2) If $M \neq 0$ and some $h \in H$ has a binding debt constraint at a node $\mu \in D(\xi)$, then $q(\xi)>0$.

Observation. Item (1) is related to the result in Santos and Woodford (1997), Theorem 3.3, that asserted that, under uniform impatience, assets in positive net supply are free of price bubbles for deflators, in the asset pricing kernel, that yield finite present values of aggregate wealth. However, we may have a positive price of money due to the presence of shadow prices in the Kuhn-Tucker deflator process (and, in this case, for any kernel deflator, the present value of aggregate wealth will be infinite).

When the portfolio constraint has a negative floor, it follows from items (1) and (2) that binding debt constraints always induce frictions, i.e. positive shadow prices. Also, if an agent becomes borrower at a certain node in $D(\xi)$, then all individuals are borrowers at some node of $D(\xi)$. In other words, in a monetary equilibrium, every agent goes short at some node.

\section{Proof of the Theorem.}

(1) By definition, if for some $h \in H,\left(\eta^{h}(\mu) ; \mu \geq \xi\right)=0$ then $F\left(\xi, q, \gamma^{h}(\xi)\right)=0$. Therefore, a monetary equilibrium is a pure bubble. However, Assumption 2 implies that bubbles are ruled out in equilibrium. Indeed, at each $\xi \in D$ there exists an agent $h=h(\xi)$ with $q(\xi) z^{h}(\xi) \geq 0$. Thus, by the impatience property, $0 \leq(1-\pi) q(\xi) z^{h}(\xi) \leq p(\xi) v(\xi)$. Moreover, this inequality and financial market feasibility allows us to find a lower bound for individual debt. Therefore, for each $h \in H$, the plan $\left(\frac{q(\xi) z^{h}(\xi)}{p(\xi) v(\xi)}\right)_{\xi \in D}$ is uniformly bounded. Furthermore, as money is in positive net supply, it follows that $\left(\frac{q(\xi)}{p(\xi) v(\xi)}\right)_{\xi \in D}$ is uniformly bounded too. Since by Proposition 2, for any $h \in H, \sum_{\xi \in D} \gamma^{h}(\xi) p(\xi) w^{h}(\xi)<+\infty$, it follows from Assumption 2 that bubbles do not arise in equilibrium.

Therefore, we conclude that, if $q(\xi)>0$ then $\left(\eta^{h}(\mu) ; \mu \geq \xi\right) \neq 0$, for all $h \in H$.

(2) Suppose that, for some $h \in H$, there exists $\mu \geq \xi$ such that that $q(\mu) z^{h}(\mu)=-p(\mu) M$. Since monotonicity of preferences implies that $p(\xi) \gg 0$, if $M \neq 0$ then $q(\mu)>0$. Also, Assumption 1 assures that Kuhn-Tucker multipliers, $\left(\gamma^{h}(\eta) ; \eta \in D\right)$, are strictly positive. Therefore, the equations that define shadow prices implies that $q(\xi)>0$. 
Some remarks,

- The proof of the Theorem implies that under Assumption 2 the monetary position of each agent is uniformly bounded - in real terms - along the even-tree. Thus, it is easy to find a vector $M^{*} \in \mathbb{R}_{+}^{L}$ such that, in any equilibrium, and for each node $\xi$, the debt constraint $q(\xi) z^{h}(\xi) \geq-p(\xi) M^{*}$ is non-binding. Therefore, when $M>M^{*}$ monetary equilibria disappear. That is, contrary to what might be expected, frictions induced by debt constraints improve welfare.

- Given a monetary equilibrium, there always exists a non-arbitrage deflator in the asset-pricing kernel and incompatible with physical Euler conditions (see Definition 2), for which the price of money is a pure bubble. Indeed, define $\nu:=(\nu(\xi): \xi \in D)$ by $\nu\left(\xi_{0}\right)=1$, and

$$
\begin{array}{rlrl}
\nu(\xi) & =1, & & \forall \xi>\xi_{0}: q(\xi)=0, \\
\frac{\nu(\xi)}{\nu\left(\xi^{-}\right)}=\frac{\gamma^{h}(\xi)}{\gamma^{h}\left(\xi^{-}\right)-\eta^{h}\left(\xi^{-}\right)}, & \forall \xi>\xi_{0}: q(\xi)>0 .
\end{array}
$$

Euler conditions on $\left(\gamma^{h}(\xi) ; \xi \in D\right)$ imply that, for each $\xi \in D, \nu(\xi) q(\xi)=\sum_{\mu \in \xi^{+}} \nu(\mu) q(\mu)$. Therefore, using the plan of deflators $\nu$, financial Euler conditions hold and the positive price of money is a bubble.

Also, since under Assumption 2 the monetary position of each agent is uniformly bounded along the event-tree, the existence of a bubble implies that under these deflators the deflated value of future individual endowments has to be infinite. This deflator is compatible with zero shadow prices and our observation conforms to the results by Santos and Woodford (1997): a monetary bubble may only occur, for a deflator in the asset pricing kernel, if for this deflator the present value of aggregate wealth is infinite.

- If we allow in our model for an increasing number of non-redundant securities in order to assure that aggregated wealth can be replicated by the deliveries of a portfolio trading plan, money will have zero price. Indeed, in this context, independently of the non-arbitrage deflator, the discounted value of future wealth must be finite (see Santos and Woodford (1997)). Therefore, if money has a positive value, we obtain a contradiction, since as we say above, we may always construct a deflator in the asset pricing kernel under which the discounted value of aggregated wealth is infinite. However, the issue of new assets, in order to achieve that efficacy of the financial markets, can be too costly.

- In models addressing the role of money as a medium of exchange, starting with Clower (1967), it is instead liquidity frictions that become crucial. In a recent work along those lines, Santos (2006) showed that monetary equilibrium only arises when cash-in-advance constraints are binding infinitely often for all agents. Also, in a cashless economy with no short-sales restrictions, Gimenez (2005) provided examples of monetary bubbles that can be reinterpreted as positive fundamental values. 
Proposition 6. Under Assumption 1, if for each $\xi \in D, u^{h}(\xi, \cdot)$ is differentiable in $\mathbb{R}_{++}^{L}$ and $\lim _{\|x\|_{m i n} \rightarrow 0^{+}} \nabla u^{h}(\xi, x)=+\infty$, then any monetary equilibrium is Pareto inefficient.

Proof. Suppose that there exists an efficient monetary equilibrium, in the sense that individuals' marginal rates of substitution coincide. As $\lim _{\|x\|_{\min \rightarrow 0^{+}}} \nabla u^{h}(\xi, x)=+\infty, \forall(h, \xi) \in H \times D$, all agents have interior consumption along the event-tree. Positive net supply of money implies that there exists, at each $\xi \in D$, at least one lender. Therefore, by the efficiency property, it follows that all individuals have zero shadow prices. Therefore, it follows from the transversality condition of Definition 2, jointly with Proposition 5, that $q(\xi)=0$ for any node $\xi \in D$. A contradiction.

\section{Monetary equilibrium in the abSence of Uniform impatience}

To highlight the role that uniform impatience has in the pricing theory of money in cashless economies, we adapt Example 1 in Araujo, Páscoa and Torres-Martínez (2007) in order to prove that without uniform impatience on preferences money may have a bubble for deflators that give a finite present value of aggregate wealth, also, even for Kuhn-Tucker multipliers. Essentially this happens because individuals will believe that, as time goes on, the probability that the economy may fall in a path in which endowments increase without an upper bound converges to zero fast enough. Notice that it must the case that the supremum over all asset pricing kernel deflators of the present value of aggregate wealth is infinite (see Santos and Woodford (1997), Theorem 3.1 and Corollary 3.2).

Example. Assume that each $\xi \in D$ has two successors: $\xi^{+}=\left\{\xi^{u}, \xi^{d}\right\}$. There are only one commodity and two agents $H=\{1,2\}$. Each $h \in H$ has physical endowments $\left(w_{\xi}^{h}\right)_{\xi \in D}$, receives financial endowments $e^{h} \geq 0$ only at the first node, and has preferences represented by the utility function $U^{h}(x)=\sum_{\xi \in D} \beta^{t(\xi)} \rho^{h}(\xi) x_{\xi}$, where $\beta \in(0,1)$ and the plan $\left(\rho^{h}(\xi)\right)_{\xi \in D} \in(0,1)^{D}$ satisfies $\rho\left(\xi_{0}\right)=1, \rho^{h}(\xi)=\rho^{h}\left(\xi^{d}\right)+\rho^{h}\left(\xi^{u}\right)$ and

$$
\rho^{1}\left(\xi^{u}\right)=\frac{1}{2^{t(\xi)+1}} \rho^{1}(\xi), \quad \rho^{2}\left(\xi^{u}\right)=\left(1-\frac{1}{2^{t(\xi)+1}}\right) \rho^{2}(\xi) .
$$

Suppose that agent $h=1$ is the only one endowed with the asset, i.e. $\left(e^{1}, e^{2}\right)=(1,0)$ and that, for each $\xi \in D$,

$$
w_{\xi}^{1}=\left\{\begin{array}{ll}
1+\beta^{-t(\xi)} & \text { if } \xi \in D^{d u}, \\
1 & \text { otherwise }
\end{array} \quad w_{\xi}^{2}= \begin{cases}1+\beta^{-t(\xi)} & \text { if } \xi \in\left\{\xi_{0}^{d}\right\} \cup D^{u d}, \\
1 & \text { otherwise }\end{cases}\right.
$$

where $D^{d u}$ is the set of nodes attained after going down followed by up, that is, $D^{d u}=\{\eta \in D$ : $\left.\exists \xi, \eta=\left(\xi^{d}\right)^{u}\right\}$ and $D^{u d}$ denotes the set of nodes reached by going up and then down, that is, $D^{u d}=\left\{\eta \in D: \exists \xi, \eta=\left(\xi^{u}\right)^{d}\right\}$.

Agents will use positive endowment shocks in low probability states to buy money and sell it later in states with higher probabilities. Let prices be $\left(p_{\xi}, q_{\xi}\right)_{\xi \in D}=\left(\beta^{t(\xi)}, 1\right)_{\xi \in D}$ and suppose that consumption of agent $h$ is given by $x_{\xi}^{h}=w_{\xi}^{h^{\prime}}$, where $h \neq h^{\prime}$. It follows from budget constraints that, at each $\xi$, the portfolio of agent $h$ must satisfy $z_{\xi}^{h}=\beta^{t(\xi)}\left(w_{\xi}^{h}-w_{\xi}^{h^{\prime}}\right)+z_{\xi^{-}}^{h}$, where $z_{\xi_{0}^{-}}^{h}:=e^{h}$ and $h \neq h^{\prime}$. 
Thus, the consumption allocations above jointly with the portfolios $\left(z_{\xi_{0}}^{1}, z_{\xi^{u}}^{1}, z_{\xi^{d}}^{1}\right)=(1,1,0)$ and $\left(z_{\xi}^{2}\right)_{\xi \in D}=\left(1-z_{\xi}^{1}\right)_{\xi \in D}$ are budget and market feasible. Finally, given $(h, \xi) \in H \times D$, let $\gamma_{\xi}^{h}=\rho^{h}(\xi)$ be the candidate for Kuhn-Tucker multiplier of agent $h$ at node $\xi$. It follows that conditions below hold and they assure individual optimality (see Proposition A2 in the Appendix A),

$$
\begin{aligned}
& \left(\gamma_{\xi}^{h} p_{\xi}, \gamma_{\xi}^{h} q_{\xi}\right)=\left(\beta^{t(\xi)} \rho^{h}(\xi), \gamma_{\xi^{u}}^{h} q_{\xi^{u}}+\gamma_{\xi^{d}}^{h} q_{\xi^{d}}\right), \\
& \sum_{\{\eta \in D: t(\eta)=T\}} \gamma_{\eta}^{h} p_{\eta} M \longrightarrow 0, \quad \text { as } T \rightarrow+\infty, \\
& \sum_{\{\eta \in D: t(\eta)=T\}} \gamma_{\eta}^{h} q_{\eta} z_{\eta}^{h} \longrightarrow 0, \quad \text { as } T \rightarrow+\infty .
\end{aligned}
$$

Note that, by construction and independently of $M \geq 0$, the plan of shadow prices associated to debt constraints is zero. Therefore, for any $M$, money has a zero fundamental value and a bubble under Kuhn-Tucker multipliers. Also, the diversity of individuals beliefs about the uncertainty (probabilities $\rho^{h}(\xi)$ ) implies that both agents perceive a finite present value of aggregate wealth. ${ }^{2}$ Finally, Assumption 2 is not satisfied, because aggregated physical endowments were unbounded along the event-tree. ${ }^{3}$

\section{Appendix}

Proof of Proposition 2. Let $\mathcal{L}_{\xi}^{h}: \mathbb{R}^{L+1} \times \mathbb{R}^{L+1} \rightarrow \mathbb{R} \cup\{-\infty\}$ be the function defined by $\mathcal{L}_{\xi}^{h}\left(y(\xi), y\left(\xi^{-}\right)\right)=$ $v^{h}(\xi, y(\xi))-\gamma^{h}(\xi) g_{\xi}^{h}\left(y(\xi), y\left(\xi^{-}\right) ; p, q\right)$, where $y(\xi)=(x(\xi), z(\xi))$ and $v^{h}(\xi, \cdot): \mathbb{R}^{L} \times \mathbb{R} \rightarrow \mathbb{R} \cup\{-\infty\}$ is given by

$$
v^{h}(\xi, y(\xi))= \begin{cases}u^{h}(\xi, x(\xi)) & \text { if } x(\xi) \geq 0 \\ -\infty & \text { otherwise }\end{cases}
$$

${ }^{2}$ Using agent' $h$ Kuhn-Tucker multipliers as deflators, the present value of aggregated wealth at $\xi \in D$, denoted by $P V_{\xi}^{h}$, satisfies,

$$
\begin{aligned}
& P V_{\xi}^{h}=\sum_{\mu \geq \xi} \frac{\gamma_{\mu}^{h}}{\gamma_{\xi}^{h}} p_{\mu} W_{\mu}=\frac{2}{\rho^{h}(\xi)} \sum_{\mu \geq \xi} \rho^{h}(\mu) \beta^{t(\mu)}+\frac{1}{\rho^{h}(\xi)} \sum_{\left\{\mu \geq \xi: \mu \in D^{\left.u d \cup D^{d u} \cup\left\{\xi_{0}^{d}\right\}\right\}}\right.} \rho^{h}(\mu)
\end{aligned}
$$

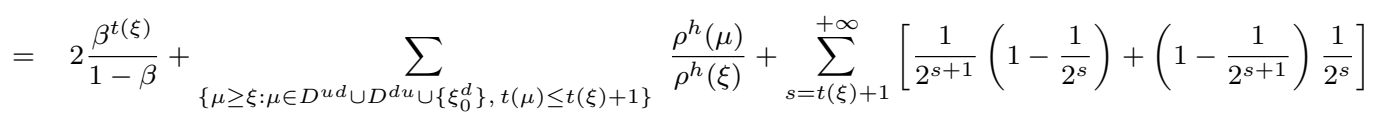

$$
\begin{aligned}
& =2 \frac{\beta^{t(\xi)}}{1-\beta}+\frac{3}{2} \frac{1}{2^{t(\xi)}}-\frac{1}{3} \frac{1}{4^{t(\xi)}}+\frac{1}{\rho^{h}(\xi)} \sum_{\left\{\mu \geq \xi: \mu \in D^{u d} \cup D^{d u}, t(\mu) \leq t(\xi)+1\right\}} \rho^{h}(\mu)<+\infty .
\end{aligned}
$$

${ }^{3}$ If Assumption B holds, there are $(\delta, \pi) \in \mathbb{R}_{++} \times(0,1)$ such that, for any $\xi \in D^{u u}:=\left\{\mu \in D: \exists \eta \in D ; \mu=\left(\eta^{u}\right)^{u}\right\}$,

$$
\frac{1}{\delta}=\frac{w_{\xi}^{h}}{\delta}>\frac{1-\pi}{\beta^{t(\xi)} \rho^{h}(\xi)} \sum_{\mu>\xi} \rho^{h}(\mu) \beta^{t(\mu)} W_{\mu}, \quad \forall h \in H .
$$

Thus, for all $(\xi, h) \in D^{u u} \times H, \beta^{t(\xi)}\left(\frac{1}{\delta(1-\pi)}+W_{\xi}\right)>P V_{\xi}^{h}$. On the other hand, given $\xi \in D^{u u}$,

$$
P V_{\xi}^{1} \geq \frac{1}{\rho^{1}(\xi)} \sum_{\left\{\mu \geq \xi: \mu \in D^{u d} \cup D^{d u}, t(\mu) \leq t(\xi)+1\right\}} \rho^{1}(\mu)=1-\frac{1}{2^{t(\xi)+1}} .
$$

Therefore, as for any $T \in \mathbb{N}$ there exists $\xi \in D^{u u}$ with $t(\xi)=T$, we conclude that, $\beta^{T}\left(\frac{1}{\delta(1-\pi)}+2\right)>0.5$, for all $T>0$. A contradiction. 
It follows from Assumption 1 and Euler conditions that, for each $T \geq 0$,

$$
\sum_{\xi \in D^{T}} \mathcal{L}_{\xi}^{h}(0,0)-\sum_{\xi \in D^{T}} \mathcal{L}_{\xi}^{h}\left(y^{h}(\xi), y^{h}\left(\xi^{-}\right)\right) \leq-\sum_{\xi \in D_{T}} \gamma^{h}(\xi) q(\xi)\left(0-z^{h}(\xi)\right) .
$$

Therefore, as for each $\xi \in D, \gamma^{h}(\xi) g_{\xi}^{h}\left(y^{h}(\xi), y^{h}\left(\xi^{-}\right) ; p, q\right)=0$, we have that, for any $S \in \mathbb{N}$,

$$
\begin{aligned}
0 \leq \sum_{\xi \in D^{S}} \gamma^{h}(\xi)\left(p(\xi) w^{h}(\xi)+q(\xi) e^{h}(\xi)\right) & \leq \limsup _{T \rightarrow+\infty} \sum_{\xi \in D^{T}} \gamma^{h}(\xi)\left(p(\xi) w^{h}(\xi)+q(\xi) e^{h}(\xi)\right) \\
& \leq U^{h}\left(x^{h}\right)+\limsup _{T} \sum_{\xi \in D_{T}} \gamma^{h}(\xi) q(\xi) z^{h}(\xi) \\
& \leq U^{h}\left(x^{h}\right)<+\infty,
\end{aligned}
$$

which concludes the proof.

Proof of Proposition 3. Suppose that $\left(y^{h}(\xi)\right)_{\xi \in D}$ is optimal for agent $h \in H$ at prices $(p, q)$. For each $T \in \mathbb{N}$, consider the truncated optimization problem,

$$
\begin{aligned}
\max & \sum_{\xi \in D^{T}} u^{h}(\xi, x(\xi)) \\
\text { s.t. } & \begin{cases}g_{\xi}^{h}\left(y(\xi), y\left(\xi^{-}\right) ; p, q\right) & \leq 0, \quad \forall \xi \in D^{T}, \text { where } y(\xi)=(x(\xi), z(\xi)), \\
q(\xi) z(\xi) & \geq-p(\xi) M, \quad \forall \xi \in D^{T} \backslash D_{T}, \\
(x(\xi), z(\eta)) & \geq 0, \quad \forall(\xi, \eta) \in D^{T} \times D_{T} .\end{cases}
\end{aligned}
$$

It follows that, under Assumption 1, each truncated problem $P^{h, T}$ has a solution $\left(y^{h, T}(\xi)\right)_{\xi \in D^{T} \cdot{ }^{4} \text { More- }}$ over, the optimality of $\left(y^{h}(\xi)\right)_{\xi \in D}$ in the original problem implies that $U^{h}\left(x^{h}\right)$ is greater than or equal to $\sum_{\xi \in D^{T}} u^{h}\left(\xi, x^{h, T}(\xi)\right)$. In fact, the plan $\left(\tilde{y}_{\xi}\right)_{\xi \in D}$ that equals to $\tilde{y}_{\xi}=y_{\xi}^{h, T}$, if $\xi \in D^{T}$, and equals to $\tilde{y}_{\xi}=0$, if $\xi \in D \backslash D_{T}$, is budget feasible in the original economy and, therefore, the allocation $\left(y^{h, T}(\xi)\right)_{\xi \in D^{T}}$ cannot improve the utility level of agent $h$.

Define $v^{h}(\xi, \cdot): \mathbb{R}^{L} \times \mathbb{R} \rightarrow \mathbb{R} \cup\{-\infty\}$ by

$$
v^{h}(\xi, y(\xi))= \begin{cases}u^{h}(\xi, x(\xi)) & \text { if } x(\xi) \geq 0 \\ -\infty & \text { in other case }\end{cases}
$$

${ }^{4}$ In fact, as $\left(y^{h}(\xi)\right)_{\xi \in D}$ is optimal and $U^{h}\left(x^{h}\right)<+\infty$, it follows that there exists a solution for $P^{h, T}$ if and only if there exists a solution for the problem,

$$
\begin{aligned}
\max & \sum_{\xi \in D^{T}} u^{h}(\xi, x(\xi)) \\
\left(\tilde{P}^{h, T}\right) & \text { s.t. } \begin{cases}g_{\xi}^{h}\left(y(\xi), y\left(\xi^{-}\right) ; p, q\right) & \leq 0, \quad \forall \xi \in D^{T}, \text { where } y(\xi)=(x(\xi), z(\xi)), \\
z(\xi) & \geq-\frac{p(\xi) M}{q(\xi)}, \forall \xi \in D^{T-1} \text { such that } q(\xi)>0 \\
z(\xi) & =0, \quad \text { if }\left[\xi \in D^{T-1} \text { and } q(\xi)=0\right] \text { or } \xi \in D_{T}, \\
x(\xi) & \geq 0, \quad \forall \xi \in D^{T} .\end{cases}
\end{aligned}
$$

Indeed, it follows from the existence of an optimal plan which gives finite utility that if $q(\xi)=0$ for some $\xi \in D$, then $q(\mu)=0$ for each successor $\mu>\xi$. Now, budget feasibility assures that,

$$
z(\xi) \leq \frac{p(\xi) w^{h}(\xi)}{q(\xi)}+z\left(\xi^{-}\right), \forall \xi \in D^{T-1} \text { such that } q(\xi)>0 .
$$

As $z\left(\xi_{0}^{-}\right)=0$, the set of feasible financial positions is bounded in the problem $\left(\tilde{P}^{h, T}\right)$. Thus, budget feasible consumption allocations are also bounded and, therefore, the set of admissible strategies is compact. As the objective function is continuous, there is a solution for $\left(\tilde{P}^{h, T}\right)$. 
where $y(\xi)=(x(\xi), z(\xi))$. Given a multiplier $\gamma \in \mathbb{R}$, let $\mathcal{L}_{\xi}^{h}(\cdot, \gamma ; p, q): \mathbb{R}^{L+1} \times \mathbb{R}^{L+1} \rightarrow \mathbb{R} \cup\{-\infty\}$ be the Lagrangian at node $\xi$, i.e.,

$$
\mathcal{L}_{\xi}^{h}\left(y(\xi), y\left(\xi^{-}\right), \gamma ; p, q\right)=v^{h}(\xi, y(\xi))-\gamma g_{\xi}^{h}\left(y(\xi), y\left(\xi^{-}\right) ; p, q\right) .
$$

It follows from Rockafellar (1997, Theorem 28.3) that there exist non-negative multipliers $\left(\gamma^{h, T}(\xi)\right)_{\xi \in D^{T}}$ such that the following saddle point property

$$
\sum_{\xi \in D^{T}} \mathcal{L}_{\xi}^{h}\left(y(\xi), y\left(\xi^{-}\right), \gamma^{h, T}(\xi) ; p, q\right) \leq \sum_{\xi \in D^{T}} \mathcal{L}_{\xi}^{h}\left(y^{h, T}(\xi), y^{h, T}\left(\xi^{-}\right), \gamma^{h, T}(\xi) ; p, q\right),
$$

is satisfied, for each plan $(y(\xi))_{\xi \in D^{T}}=(x(\xi), z(\xi))_{\xi \in D^{T}}$ for which

$$
\begin{array}{rlrl}
(x(\xi), z(\eta)) & \geq 0, & & \forall(\xi, \eta) \in D^{T} \times D_{T}, \\
q(\xi) z(\xi) & \geq-p(\xi) M, & \forall \xi \in D^{T} \backslash D_{T} .
\end{array}
$$

Moreover, at each node $\xi \in D^{T}$, multipliers satisfy $\gamma^{h, T}(\xi) g_{\xi}^{h}\left(y^{h, T}(\xi), y^{h, T}\left(\xi^{-}\right) ; p, q\right)=0$.

Analogous arguments to those made in Claims A1-A3 in Araujo, Páscoa and Torres-Martínez (2007) implies that,

ClAIM. Under Assumption 1, the following conditions hold:

(i) For each $t<T$,

$$
0 \leq \sum_{\xi \in D^{t}} \gamma^{h, T}(\xi)\left(p(\xi) w^{h}(\xi)+q(\xi) e^{h}(\xi)\right) \leq U^{h}\left(x^{h}\right)
$$

(ii) For each $0<t<T$,

$$
\sum_{\xi \in D_{t}} \gamma^{h, T}(\xi) q(\xi) z^{h}\left(\xi^{-}\right) \leq \sum_{\xi \in D \backslash D^{t-1}} u^{h}\left(\xi, x^{h}(\xi)\right)
$$

(iii) For each $\xi \in D^{T-1}$ and for any $y(\xi)=(x(\xi), z(\xi))$, with $x(\xi) \geq 0$ and $q(\xi) z(\xi) \geq-p(\xi) M$,

$$
\begin{array}{r}
u^{h}(\xi, x(\xi))-u^{h}\left(\xi, x^{h}(\xi)\right) \leq\left(\gamma^{h, T}(\xi) p(\xi) ; \gamma^{h, T}(\xi) q(\xi)-\sum_{\mu \in \xi^{+}} \gamma^{h, T}(\mu) q(\mu)\right) \cdot\left(y(\xi)-y^{h}(\xi)\right) \\
+\sum_{\eta \in D \backslash D^{T}} u^{h}\left(\eta, x^{h}(\eta)\right) .
\end{array}
$$

Now, at each $\xi \in D, \underline{w}^{h}(\xi):=\min _{l \in L} w^{h}(\xi, l)>0$. Also, as a consequence of monotonicity of $u^{h}(\xi)$, $\|p(\xi)\|_{\Sigma}>0$. Thus, item (i) above guarantees that, for each $\xi \in D$,

$$
0 \leq \gamma^{h, T}(\xi) \leq \frac{U^{h}\left(x^{h}\right)}{\underline{w}^{h}(\xi)\|p(\xi)\|_{\Sigma}}, \quad \forall T>t(\xi) .
$$

Therefore, the sequence $\left(\gamma^{h, T}(\xi)\right)_{T \geq t(\xi)}$ is bounded, node by node. As the event-tree is countable, there is a common subsequence $\left(T_{k}\right)_{k \in \mathbb{N}} \subset \mathbb{N}$ and non-negative multipliers $\left(\gamma^{h}(\xi)\right)_{\xi \in D}$ such that, for each $\xi \in D$, $\gamma^{h, T_{k}}(\xi) \rightarrow_{k \rightarrow+\infty} \gamma^{h}(\xi)$, and

$$
\begin{aligned}
\gamma^{h}(\xi) g_{\xi}^{h}\left(p, q, y^{h}(\xi), y^{h}\left(\xi^{-}\right)\right) & =0 \\
\limsup _{t \rightarrow+\infty} \sum_{\xi \in D_{t}} \gamma^{h}(\xi) q(\xi) z^{h}\left(\xi^{-}\right) & \leq 0
\end{aligned}
$$

where equation (2) follows from the strictly monotonicity of $u^{h}(\xi)$, and equation (3) is a consequence of item (ii) (taking the limit as $T$ goes to infinity and, afterwards, the limit in $t$ ). 
Moreover, using item (iii), and taking the limit as $T$ goes to infinity, we obtain that, for each $y(\xi)=$ $(x(\xi), z(\xi))$, with $x(\xi) \geq 0$ and $q(\xi) z(\xi) \geq-p(\xi) M$,

$$
u^{h}(\xi, x(\xi))-u^{h}\left(\xi, x^{h}(\xi)\right) \leq\left(\gamma^{h}(\xi) p(\xi) ; \gamma^{h}(\xi) q(\xi)-\sum_{\mu \in \xi^{+}} \gamma^{h}(\mu) q(\mu)\right) \cdot\left(y(\xi)-y^{h}(\xi)\right) .
$$

Let $\mathcal{F}^{h}(\xi, p, q)=\left\{(x, z) \in \mathbb{R}^{L} \times \mathbb{R}: x \geq 0 \wedge q(\xi) z \geq-p(\xi) M\right\}$.

It follows that $\left(\gamma^{h}(\xi) p(\xi) ; \gamma^{h}(\xi) q(\xi)-\sum_{\mu \in \xi^{+}} \gamma^{h}(\mu) q(\mu)\right)$ belongs to the super-differential set of the function $v^{h}(\xi, \cdot)+\delta\left(\cdot, \mathcal{F}^{h}(\xi, p, q)\right)$ at point $y^{h}(\xi)$, where $\delta\left(y, \mathcal{F}^{h}(\xi, p, q)\right)=0$, when $y \in \mathcal{F}^{h}(\xi, p, q)$ and $\delta\left(y, \mathcal{F}^{h}(\xi, p, q)\right)=-\infty$, otherwise. Notice that, for each $y \in \mathcal{F}^{h}(\xi, p, q), \kappa \in \partial \delta\left(y, \mathcal{F}^{h}(\xi, p, q)\right) \Leftrightarrow 0 \leq$ $k\left(y^{\prime}-y\right), \forall y^{\prime} \in \mathcal{F}^{h}(\xi, p, q)$.

Now, by Theorem 23.8 in Rockafellar (1997), for all $y \in \mathcal{F}^{h}(\xi, p, q)$, if $v^{\prime}(\xi)$ belongs to $\partial\left[v^{h}(\xi, y)+\delta\left(y, \mathcal{F}^{h}(\xi, p, q)\right)\right]$ then there exists $\tilde{v}^{\prime}(\xi) \in \partial v^{h}(\xi, y)$ such that both $v^{\prime}(\xi) \geq \tilde{v}^{\prime}(\xi)$ and $\left(v^{\prime}(\xi)-\right.$ $\left.\tilde{v}^{\prime}(\xi)\right) \cdot(x, q(\xi) z+p(\xi) M)=0$, where $y=(x, z)$. Therefore, it follows that there exists, for each $\xi \in D$, a super-gradient $\tilde{v}^{\prime}(\xi) \in \partial v^{h}\left(\xi, y^{h}(\xi)\right)$ such that,

$$
\begin{array}{r}
\left(\gamma^{h}(\xi) p(\xi) ; \gamma^{h}(\xi) q(\xi)-\sum_{\mu \in \xi^{+}} \gamma^{h}(\mu) q(\mu)\right)-\tilde{v}^{\prime}(\xi) \geq 0, \\
{\left[\left(\gamma^{h}(\xi) p(\xi) ; \gamma^{h}(\xi) q(\xi)-\sum_{\mu \in \xi^{+}} \gamma^{h}(\mu) q(\mu)\right)-\tilde{v}^{\prime}(\xi)\right] \cdot\left(x^{h}(\xi), q(\xi) z^{h}(\xi)+p(\xi) M\right)=0 .}
\end{array}
$$

As $\tilde{v}^{\prime}(\xi) \in \partial v^{h}\left(\xi, y^{h}(\xi)\right)$ if and only if there is $u^{\prime}(\xi) \in \partial u^{h}\left(\xi, x^{h}(\xi)\right)$ such that $\tilde{v}^{\prime}(\xi)=\left(u^{\prime}(\xi), 0\right)$, it follows from last inequalities that Euler conditions hold.

On the other side, item (i) in claim above guarantees that, $\sum_{\xi \in D} \gamma^{h}(\xi)\left(p(\xi) w^{h}(\xi)+q(\xi) e^{h}(\xi)\right)<+\infty$ and, therefore, equations (2) and (3) assure that,

$$
\begin{aligned}
\limsup _{t \rightarrow+\infty} \sum_{\xi \in D_{t}} \gamma^{h}(\xi) q(\xi) z^{h}(\xi) & \leq \limsup _{t \rightarrow+\infty} \sum_{\xi \in D_{t}} \gamma^{h}(\xi)\left(p(\xi) w^{h}(\xi)+q(\xi) e^{h}(\xi)+q(\xi) z^{h}\left(\xi^{-}\right)\right) \\
& \leq \limsup _{t \rightarrow+\infty} \sum_{\xi \in D_{t}} \gamma^{h}(\xi) q(\xi) z^{h}\left(\xi^{-}\right) \leq 0
\end{aligned}
$$

which implies that transversality condition holds.

Proof of Proposition 4. Note that, under the hypotheses in the proposition, it follows that

$$
\lim _{T \rightarrow+\infty} \sum_{\xi \in D_{T}} \gamma^{h}(\xi) q(\xi) z^{h}(\xi)=0
$$

On the other hand, it follows from Euler conditions that, for each $T \geq 0$,

$$
\sum_{\xi \in D^{T}} \mathcal{L}_{\xi}^{h}\left(y(\xi), y\left(\xi^{-}\right), \gamma^{h}(\xi) ; p, q\right)-\sum_{\xi \in D^{T}} \mathcal{L}_{\xi}^{h}\left(y^{h}(\xi), y^{h}\left(\xi^{-}\right), \gamma_{\xi}^{h} ; p, q\right) \leq-\sum_{\xi \in D_{T}} \gamma^{h}(\xi) q(\xi)\left(z(\xi)-z^{h}(\xi)\right)
$$

Moreover, as at each node $\xi \in D$ we have that $\gamma^{h}(\xi) g_{\xi}^{h}\left(y^{h}(\xi), y^{h}\left(\xi^{-}\right) ; p, q\right)=0$, each budget feasible allocation $y=((x(\xi), z(\xi)) ; \xi \in D)$ must satisfy

$$
\sum_{\xi \in D^{T}} u^{h}(\xi, x(\xi))-\sum_{\xi \in D^{T}} u^{h}\left(\xi, x^{h}(\xi)\right) \leq-\sum_{\xi \in D_{T}} \gamma^{h}(\xi) q(\xi)\left(z(\xi)-z^{h}(\xi)\right)
$$




$$
\begin{aligned}
& \text { Now, as the sequence }\left(\sum_{\xi \in D_{T}} \gamma^{h}(\xi) q(\xi) z^{h}(\xi)\right)_{T \in \mathbb{N}} \text { converges, it is bounded. Thus, } \\
& \qquad \begin{aligned}
\limsup _{T \rightarrow+\infty}\left(-\sum_{\xi \in D_{T}} \gamma^{h}(\xi) q(\xi)\left(z(\xi)-z^{h}(\xi)\right)\right) & \leq \limsup _{T \rightarrow+\infty}\left(-\sum_{\xi \in D_{T}} \gamma^{h}(\xi) q(\xi) z(\xi)\right) \\
& \leq \lim _{T \rightarrow+\infty} \sum_{\xi \in D_{T}} \gamma^{h}(\xi) p(\xi) M=0 .
\end{aligned}
\end{aligned}
$$

Therefore,

$$
U^{h}(x)=\limsup _{T \rightarrow+\infty} \sum_{\xi \in D^{T}} u^{h}(\xi, x(\xi)) \leq U^{h}\left(x^{h}\right)
$$

which guarantees that the allocation $\left(x^{h}(\xi), z^{h}(\xi)\right)_{\xi \in D}$ is optimal.

Proof of Proposition 5. By Proposition 3, there are, for each agent $h \in H$, non-negative shadow prices $\left(\eta^{h}(\xi) ; \xi \in D\right)$, satisfying for each $\xi \in D$,

$$
\begin{aligned}
0 & =\eta^{h}(\xi)\left(q(\xi) z^{h}(\xi)+p(\xi) M\right) \\
\gamma^{h}(\xi) q(\xi) & =\sum_{\mu \in \xi^{+}} \gamma^{h}(\mu) q(\mu)+\eta^{h}(\xi) q(\xi) .
\end{aligned}
$$

Therefore,

$$
\gamma^{h}(\xi) q(\xi)=\sum_{\mu \geq \xi} \eta^{h}(\mu) q(\mu)+\lim _{T \rightarrow+\infty} \sum_{\mu \in D_{T}(\xi)} \gamma^{h}(\mu) q(\mu)
$$

As multipliers and monetary prices are non-negative, the infinite sum in the right hand side of equation above is well defined, because its partial sums are increasing and bounded by $\gamma^{h}(\xi) q(\xi)$. This also implies that the limit of the (discounted) asset price exists.

\section{REFERENCES}

[1] Araujo, A., J. Fajardo, and M.R. Páscoa (2005): "Endogenous collateral," Journal of Mathematical Economics, 41, 439-462.

[2] Araujo, A., M.R. Páscoa, and J.P. Torres-Martínez (2007): "Long-lived Collateralized Assets and Bubbles," Working paper No 542, PUC-Rio, Brazil. Available at http://www.econ.puc-rio.br/PDF/td542.pdf

[3] Bewley, T. (1980): "The Optimal Quantity of Money," in Models of Monetary Economics, ed. by J. Kareken and N. Wallace. Minneapolis: Federal Reserve Bank.

[4] Clower, R. (1967): "A Reconsideration of the Microfundations of Monetary Theory," Western Economic Journal $6,1-8$.

[5] Gimenez, E. (2005): "On the Positive Fundamental Value of Money with Short-Sale Constraints: A Comment on Two Examples," Annals of Finance, forthcoming.

[6] Hernandez, A., and M. Santos (1996): "Competitive Equilibria for Infinite-Horizon Economies with Incomplete Markets," Journal of Economic Theory, 71, 102-130.

[7] ouini, E., and H. Kallal (1995): "Arbitrage in security markets with short-sales constraints," Mathematical Finance, 5 197-232.

[8] Laibson, D. (1998): "Life-cicle consumption and hyperbolic discount functions," European Economic Review, $42,861-871$

[9] Magill, M., and M. Quinzii (1996): "Incomplete Markets over an Infinite Horizon: Long-lived Securities and Speculative Bubbles," Journal of Mathematical Economics, 26, 133-170.

[10] Ricón-Zapatero, J.P., and M. Santos (2007): "Differentiability of the Value Function without Interiority Assumptions," working paper 07-14, Ec. Series 05, U. Carlos III de Madrid. 
[11] Samuelson, P. (1958): "An Exact Consumption-Loan Model of Interest with or without the Social Contrivance of Money," Journal of Political Economy, 66, 467-482.

[12] Santos, M. (2006): "The Value of Money in a Dynamic Equilibrium Model," Economic Theory, 27, 39-58.

[13] Santos, M., and M. Woodford (1997): "Rational Asset Pricing Bubbles," Econometrica, 65, 19-57.

[14] Rockafellar, R.T. (1997): "Convex analysis," Princeton University Press, Princeton, New Jersey, USA.

Faculdade de Economia, Universidade Nova de Lisboa

Travessa Estevão Pinto, 1099-032 Lisbon, Portugal.

E-mail address: pascoa@fe.unl.pt

Central Bank of Brazil and Department of Economics, PUC-Rio

Rua Marquês de São Vicente 225, Gávea, 22453-900 Rio de Janeiro, Brazil.

E-mail address: myrian@econ.puc-rio.br

Department of Economics, University of Chile

Diagonal Paraguay 257, office 1604, Santiago, Chile.

E-mail address: jptorres.martinez@gmail.com 